\title{
Cystathionine $\beta$-synthase deficiency: Effects of betaine supplementation after methionine restriction in B6-nonresponsive homocystinuria
}

Rani H. Singh, $P h D^{1}$, Warren D. Kruger, PhD', Liqun Wang, $P h D^{2}$, Marzia Pasquali, PhD ${ }^{3}$, and Louis J. Elsas II, MD

\begin{abstract}
Purpose: For treatment of cystathionine $\beta$-synthase $(C \beta S)$ deficiency, we determined the effect of betaine ( $N, N, N$-trimethylglycine) therapy and examined the genotype-phenotype relationships to betaine. Methods: In five patients with B6-nonresponsive homocystinuria, we defined the $\mathrm{C} \beta S$ genotypes and determined metabolic responses to betaine as an additive to traditional dietary methionine restriction. Results: After betaine therapy, thcy declined (mean $47.4 \mu \mathrm{mol} / \mathrm{L}$; range: -21.2 to $-104.0 \mu \mathrm{mol} / \mathrm{L} ; P=0.02$ ), whereas total plasma cysteine and methionine did not change. Plasma methionine/tHcy ratios increased by 5.45 (range: +1.5 to $15.3 ; P=0.05$ ) inpatients with B6-nonresponsive alleles. Conclusion: Betaine improves metabolic control in B6-nonresponsive patients with homocystinuria after optimum dietary control. Genet Med 2004:6(2):90-95.
\end{abstract}

Key Words: betaine therapy, B6-nonresponsive, cystathionine $\beta$-synthase deficiency, genotype-phenotype correlation, homocystinuria

Homocystinuria caused by cystathionine $\beta$-synthase (C $\beta S$, EC no. 4.2.1.22) deficiency is an inborn error of sulfur amino acid metabolism. Worldwide, estimates of the incidence of C $\beta$ S deficiency range from $1 / 20,500$ in Denmark ${ }^{1}$ to $1 / 800,000$ in Japan. ${ }^{2} \mathrm{C} \beta S$, the first enzyme in the transsulfuration pathway, catalyzes the condensation of homocysteine and serine to produce cystathionine, and ultimately, cysteine (Fig. 1). C $\beta S$ uses pyridoxal phosphate (B6) and heme as its cofactors. ${ }^{3,4}$

Total plasma homocysteine (tHcy) is markedly elevated in $\mathrm{C} \beta \mathrm{S}$ deficiency and is a sensitive in vivo marker for therapeutic intervention. ${ }^{2}$ Even mild to moderate elevation of tHcy is a risk-factor for premature vascular disease (PVD), much like cholesterol.5,6 Normalization of tHcy is sought in persons with $\mathrm{C} \beta \mathrm{S}$ deficiency in order to lower their lifetime risk for PVD. ${ }^{7}$ However, a portion of patients with $C \beta S$ deficiency, even during optimal dietary compliance, can not lower tHcy to the normal range (personal observations and Table 2).

Therapeutically, patients with $\mathrm{C} \beta \mathrm{S}$ deficiency can be divided into two groups, based upon the effects of pharmacological pyridoxine therapy on their metabolic status and clinical outcome. Patients experiencing significant decreases in both plasma methi-

\footnotetext{
From the ${ }^{1}$ Division of Medical Genetics, Department of Human Genetics, Emory University School of Medicine, Atlanta, Georgia; ${ }^{2}$ Division of Population Science, Fox Chase Cancer Center, Philadelphia, Pennsylvania; ${ }^{3}$ Department of Pathology, University of Utah, Salt Lake City, Utah; and ${ }^{4}$ Dr. John T. Macdonald Foundation Center for Medical Genetics, University of Miami School of Medicine, Miami, Florida.

Rani H. Singh, PhD, RD, Division of Medical Genetics, Department of Human Genetics, Emory University School of Medicine, 2040 Ridgewood Drive, Atlanta, GA 30322.

Received: October 1, 2003

Accepted: December 8, 2003.
}

DOI: 10.1097/01.GIM.0000117334.84388.F4 onine and free plasma homocystine (fHcys) while receiving pharmacological amounts of pyridoxine without diet manipulation are considered "B6-responsive." Patients showing no significant decreases in methionine or fHys while receiving up to $1.0 \mathrm{~g} /$ day of pyroxidine following pyroxidine are classified as "B6-nonresponsive." ${ }^{2,8-11}$ Such individuals demonstrate minimal or absent residual $C \beta S$ activity in cultured fibroblasts. ${ }^{2,8-11}$

Betaine ( $N, N, N$-trimethylglycine) is a natural product of choline catabolism and serves as a methyl-donor for homocysteine to synthesize methionine by betaine-homocysteine S-methyltransferase (BHMT, EC 2.1.1.5) (Fig. 1). This enzyme, present primarily in the liver and kidney, is induced by betaine and can remethylate up to $25 \%$ of total homocysteine flux. ${ }^{12}$

Betaine improved biochemical control in previous studies of patients with B6-nonresponsive C $\beta$ S deficiency. ${ }^{13}$ Subsequent studies confirmed their observations that betaine treatment decreased total plasma homocysteine, in both plasma and cerebrospinal fluid. ${ }^{9,14,15}$ However, because standardized dietary management did not precede the use of betaine in these studies, the additive effect of betaine to "dietary control" remained unknown. In this study, our patients achieved their optimal dietary control, based upon their nutritional history and metabolic measures of plasma methionine and tHcy. We then assessed the additive effect of betaine on $\mathrm{tHcy}$ and plasma methionine. We determined their molecular genotypes for $\mathrm{C} \beta \mathrm{S}$ deficiency and correlated them with ethnicity and clinical B6 nonresponsivity.

\section{MATERIALS AND METHODS}

\section{Patient recruitment}

Five patients with biochemically confirmed cystathionine $\beta$-synthase deficiency cared for by the Division of Medical Ge- 


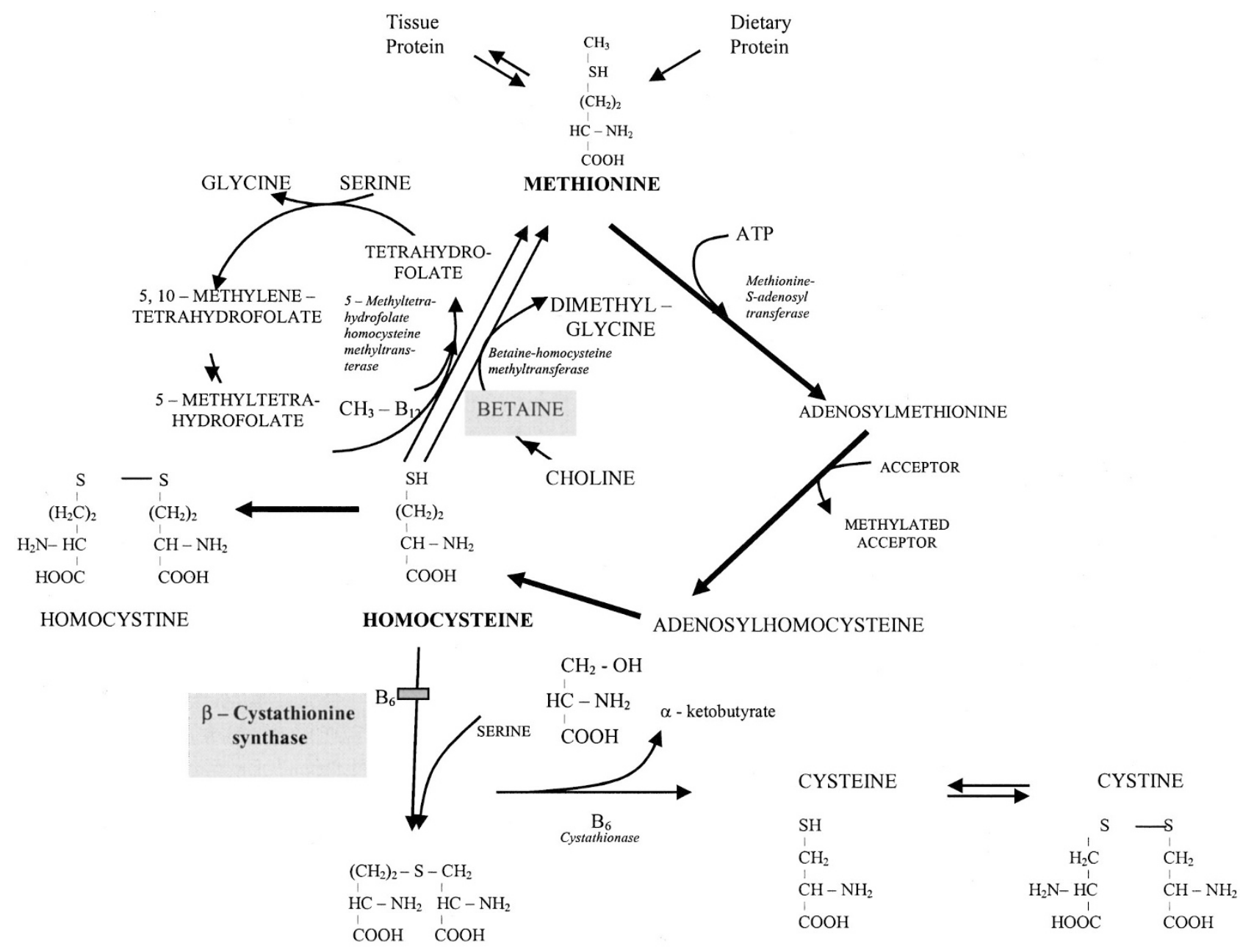

CYSTATHIONINE

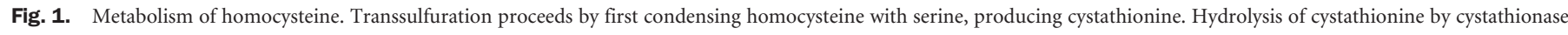

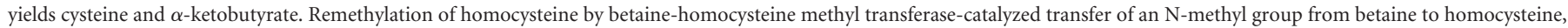
yielding methionine and $N, N$-dimethylglycine.

netics at Emory University were recruited for this study. The study and its informed consent were approved by Emory University's Human Investigations Committee. Diagnoses were established by elevated methionine, free homocystine, and decreased free cystine for patients diagnosed before 1996. Total plasma homocysteine quantitation began in 1996. Molecular genotyping of $\mathrm{C} \beta \mathrm{S}$ confirmed these biochemical diagnoses in all patients. Three of the five patients were identified by positive newborn screening for hypermethioninemia, and two were identified later in life after the discovery of ectopia lentis. All patients required methionine restriction to $<30 \mathrm{mg} / \mathrm{kg}$ per day, to decrease their free homocystine and total plasma homocysteine concentrations. Methionine-restricted diets were implemented concurrently with pyridoxine supplementation at doses up to $20 \mathrm{mg} / \mathrm{kg}$ per day. We selected for this study five patients classified as B6-nonresponsive. All were treated with methionine restriction and pharmacological supplementation of pyridoxine, but could not maintain plasma methionine be- low $50 \mu \mathrm{mol} / \mathrm{L}$ or free homocystine below $5 \mu \mathrm{mol} / \mathrm{L}$. The treatment of B6-nonresponsive C $\beta$ S deficiency patients with "methionine restriction" was accomplished in part by the simultaneous dietary restriction of natural protein, and supplementation with medical foods free of methionine. These methionine-free medical foods were supplemented with conditionally-essential L-cysteine, in the form of L-cystine.

\section{Study design}

Patients were first treated with natural protein restriction, supplementation with methionine-depleted, L-cystine-fortified medical foods, and 5 to $20 \mathrm{mg} / \mathrm{kg}$ per day of pyridoxine. ${ }^{16}$ Upon attaining the lowest plasma methionine and total plasma homocysteine, we began betaine at prescribed doses of $20-50$ $\mathrm{mg} / \mathrm{kg}$ per day. Dosages were increased to $120-150 \mathrm{mg} / \mathrm{kg}$ per day and provided as three divided doses until stabilization of tHcy to lowest concentrations for the individual. Biochemical measurements were obtained every one to three months, until 
the tHcy reached a nadir. Three-day diet histories were analyzed and compared for methionine content, and other essential nutrients throughout the studies.

\section{Statistical analyses}

The Student's $t$ test was used for comparisons of each metabolic parameter in each patient before and during betaine therapy. Significance was reported for $P \leq 0.05$.

\section{Biochemical measurements}

Total plasma homocysteine and total plasma cysteine concentrations were determined after chemical reduction, derivitization with a fluorophore, and subsequent HPLC separation as previously described. ${ }^{17}$ Plasma amino acid concentrations (including methionine and free homocystine) were determined by traditional methods of ion-exchange chromatography. ${ }^{18}$

\section{Molecular analyses of the $\mathrm{C} \beta S$ gene}

Nucleic Acids were isolated from $75 \mathrm{~mL}$ of cells using the Purescript RNA Isolation kit (Gentra Systems, Minneapolis, $\mathrm{MN}$ ). The fifteen coding exons of $\mathrm{C} \beta \mathrm{S}$ were amplified using the primer pairs described in Table $1 .{ }^{19}$ After PCR the products were isolated by gel purification using the QIAEXII Gel Extraction Kit (Qiagen, Valencia, CA) and sequenced at the Fox Chase Cancer Center. Mutant alleles were tested individually in a yeast functional assay as previously described. ${ }^{20,21}$ All 6 mutant alleles identified in these 5 patients failed to augment cysteine auxotrophy consistent with these mutations affecting enzyme function, as shown in Table 2.

\section{RESULTS}

\section{Genotypes of patients studied}

The molecular bases for the five patients studied with clinical B6-nonresponsive C $\beta$ S deficiency are shown in Table 2. Two Caucasian patients (patients 4 and 3) were compound heterozygotes for the common European I278T mutation, an L101P alteration observed in several Irish patients, and a novel D376N mutation, respectively. ${ }^{22,23}$ One Caucasian patient ( $\mathrm{Pa}-$ tient 2) was homozygous for a V320A mutation previously observed in a single Norwegian patient. ${ }^{24}$ Two American Black patients (patients 5 and 1) possessed a T353M allele. ${ }^{25,26}$ One (Patient 5) was homozygous for this mutation, and the other (Patient 1) was compound heterozygous for this mutation and a novel Q526K mutation. All patients were classified as B6nonresponsive by clinical criteria.

\section{Effect of betaine on plasma methionine}

Table 3 shows the plasma methionine concentrations obtained immediately before the initiation of betaine but after optimum B6 and dietary therapy. The listed plasma methionine concentrations $(\mu \mathrm{mol} / \mathrm{L})$ reflect the effect of betaine therapy. Plasma methionine concentrations increased about 2 -fold in patients 2,3 , and 5 , and 4 -fold in patient 1 . None of these patients reported a change in methionine dietary intake (data not shown). Plasma methionine concentration decreased
Table 1

Oligonucleotide primer sequences used to sequence the sixteen exons of the cystathionine $\beta$-synthase gene

\begin{tabular}{|c|c|c|}
\hline Exon & Primers & $\begin{array}{l}\text { PCR fragment size } \\
\text { (bp) }\end{array}$ \\
\hline \multirow[t]{2}{*}{1} & $5^{\prime}$-ttcgctggaaccccacagca-3' & 344 \\
\hline & $5^{\prime}$-tgtccaggtaacaaactcctg- $3^{\prime}$ & \\
\hline \multirow[t]{2}{*}{2} & $5^{\prime}$-tctgccagggctggtactat- $3^{\prime}$ & 283 \\
\hline & $5^{\prime}$-ctgatcccagggccttgcct- $3^{\prime}$ & \\
\hline \multirow[t]{2}{*}{3} & $5^{\prime}$-gggtgagcaggaatcaatgg-3' & 270 \\
\hline & $5^{\prime}$-tcccggcaggctcggcatgg-3' & \\
\hline \multirow[t]{2}{*}{$4+5+6$} & $5^{\prime}$-cagggcttggggggtcactg-3' & 592 \\
\hline & $5^{\prime}$-ctcccaggcagccagggata-3' & \\
\hline \multirow[t]{2}{*}{7} & $5^{\prime}$-gaactttttggttacccacc-3' & 533 \\
\hline & $5^{\prime}$-caagccccagttgaggggca-3' & \\
\hline \multirow[t]{2}{*}{8} & $5^{\prime}$-gaatatcgaggcatgtccag-3' & 332 \\
\hline & $5^{\prime}$-cagcttctcaccatgcgtgc- $3^{\prime}$ & \\
\hline \multirow[t]{2}{*}{9} & $5^{\prime}$-ggctgttcaccctcttggtc- $3^{\prime}$ & 208 \\
\hline & $5^{\prime}$-gtgccccctagccatctctg-3' & \\
\hline \multirow[t]{2}{*}{10} & $5^{\prime}$-gtgcacaattcatgcatacg- $3^{\prime}$ & 170 \\
\hline & $5^{\prime}$-ggtgaggcgtgagaggcatc- $3^{\prime}$ & \\
\hline \multirow[t]{2}{*}{$11+12$} & $5^{\prime}$-gcgtgccactcagcaggggc- $3^{\prime}$ & 597 \\
\hline & $5^{\prime}$-cctgtccagtgacactgacg- $3^{\prime}$ & \\
\hline \multirow[t]{2}{*}{13} & $5^{\prime}$-gcagaggacttccatgtgtg- $3^{\prime}$ & 296 \\
\hline & $5^{\prime}$-ccgggcacctgtttgagctg- $3^{\prime}$ & \\
\hline \multirow[t]{2}{*}{14} & $5^{\prime}$-gcaggacccaccatcgcatc- $3^{\prime}$ & 241 \\
\hline & $5^{\prime}$-catggcagaggccaggcttg- $3^{\prime}$ & \\
\hline \multirow[t]{2}{*}{16} & $5^{\prime}$-ccacccagcctcccacggca- $3^{\prime}$ & 231 \\
\hline & $5^{\prime}$-gtttagggctcaggaaagcg-3' & \\
\hline
\end{tabular}

Table 2

Human $\mathrm{C} \beta \mathrm{S}$ activity determined in total yeast extracts

\begin{tabular}{lcc}
\hline Amino acid substitution & $\begin{array}{c}\mathrm{C} \beta \text { S activity } \\
(\text { nmol per hr) }\end{array}$ & $\begin{array}{c}\text { C } \beta \text { S activity } \\
\text { (\% of wild type) }\end{array}$ \\
\hline T353M & $<10$ & $<1.7 \%$ \\
Q526K & 26 & $4.4 \%$ \\
V320A & 213 & $36 \%$ \\
I278T & 14 & $2.4 \%$ \\
D376N & 10 & $<1.7 \%$ \\
L101P & $<10$ & $<1.0 \%$ \\
\hline
\end{tabular}

${ }^{a}$ Assays were performed twice using methods previously described $\left.{ }^{20}\right)$. The average is given, and the standard deviation for all samples was $<30$.

about $16 \%$ in patient 4 , who reported a decrease in methionine intake, increased compliance with the metabolic formula, and inconsistent compliance with the prescribed betaine dosing regimen (discussed later). 


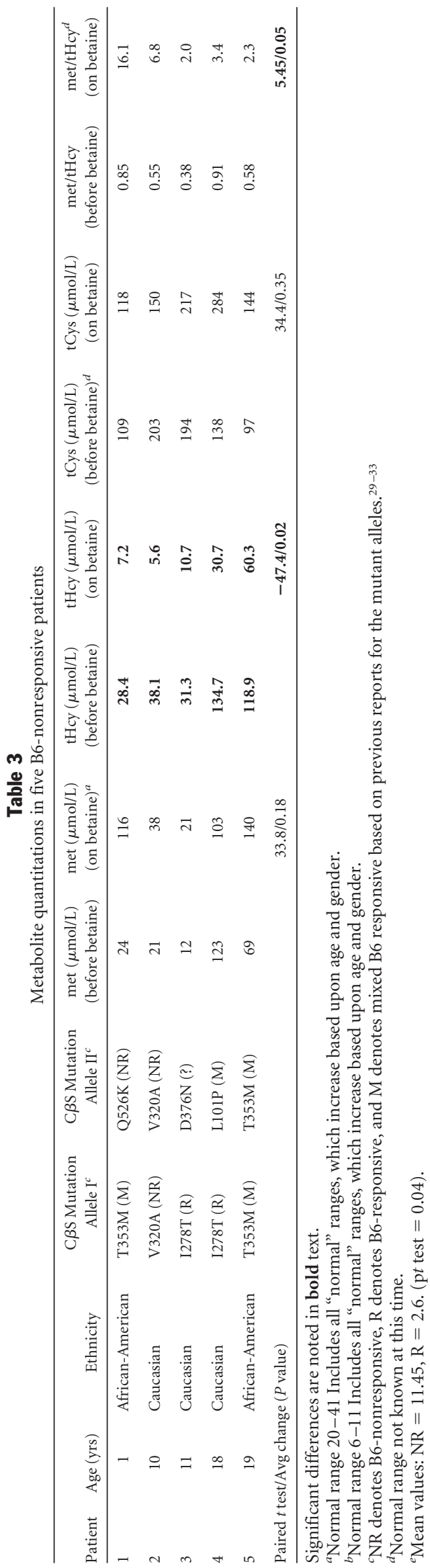

\section{Effect of betaine on total plasma homocysteine}

Total plasma homocysteine concentrations obtained before initiation of betaine therapy, and after maximal therapy, are shown in Table 2. All patients experienced significant reductions in tHcy, regardless of genotype or plasma methionine concentrations. Patients 1, 2, and 3 normalized their total plasma homocysteine concentrations on betaine therapy, despite elevated concentrations before the initiation of betaine. Even patients 4 and 5, who are adolescents with variable compliance to diet, demonstrated decreased tHcy concentrations.

\section{Effect of betaine on total plasma cysteine}

Total plasma cysteine concentrations did not significantly change before and during betaine therapy in the group as a whole (Table 3). Although Patient 4 experienced a doubling of total plasma cysteine concentrations during betaine therapy, he also reported increased intake of cystine-fortified medical food.

\section{Effect of betaine on the ratio of methionine/total plasma homocysteine}

Because betaine decreases homocysteine and increases methionine by providing an additional pathway for homocysteine transmethylation to methionine, a ratio of this substrate and product were good biochemical parameters of response to betaine (Table 3). During betaine addition, all patients experienced an increase in the ratio of plasma methionine/tHcy, regardless of estimated methionine intakes, compliance with methionine restriction or betaine. As seen by comparing the methionine/tHcy ratios in Table 2, even Patient 4 had an approximately 4 -fold increase in this ratio when betaine was added to his therapeutic regimen.

\section{DISCUSSION}

Several previous publications reported the effective use of betaine in $\mathrm{C} \beta \mathrm{S}$ deficiency. Most groups noted improvement in metabolic control. ${ }^{9} 13,27,28$ However, genotype-phenotype relationships, the effects on total plasma homocysteine concentrations (tHcy), the additive effect after dietary control, and the ratio of methionine to tHcy were not reported. In their pioneer work, Smolin et al. ${ }^{29}$ first demonstrated the clinical efficacy of betaine, and a later study by Wilcken et al. ${ }^{13}$ found that betaine decreased estimated tHcy and increased estimated tCys, which were determined from free homocystine and free homocysteine-cysteine mixed disulfide concentrations. This study also found variable effects of betaine on plasma methionine concentrations. In subsequent studies, when tHcy was measured in patients with $C \beta S$ deficiency, betaine decreased total plasma homocysteine concentrations in both plasma and cerebrospinal fluid. ${ }^{9}, 14$ These more recent studies showed the efficacy of betaine and supported its approval by the FDA as an "orphan drug" for the treatment of homocystinuria.

We observed significant and clinically relevant "net" metabolic effects of betaine in patients with B6-nonresponsive C $\beta S$ 
deficiency. The response to betaine was greatest among patients 1 and 2 who had mutations previously described as B6nonresponsive (NR) $25,24,30$ compared to patients who were heterozygous for mutant alleles previously defined as B6-responsive. ${ }^{31-33}$ The dramatic falls in total plasma homocysteine in all 5 patients were reflected in the mean decrease of over 47.4 $\mu \mathrm{mol} / \mathrm{L}$. Although we observed $4 / 5$ patients with a near doubling of their plasma methionine during maximal betaine therapy, the mean plasma methionine in our betaine treatment group did not change. This was because of the decrease in the plasma methionine of patient 4 , for which we found two contributing explanations. First, this patient reported improved compliance with both methionine restriction and consumption of the methionine-depleted, cystine-enriched medical food. Objective evidence supporting improved compliance by Patient 4 was the doubling of his total plasma cysteine and the decrease in plasma methionine during betaine treatment, which was only observed in this subject. This patient also reported being partially compliant with his betaine dosing regimen, only taking one fourth of the prescribed dose, resulting in an intake of approximately 20 to $30 \mathrm{mg} / \mathrm{kg}$ per day. Therefore, with both decreased methionine intake and suboptimal betaine dosing, it is not surprising that his plasma methionine decreased compared to baseline when all other patients' plasma methionines increased after betaine therapy. Of interest was that his ratio of met/tHcy increased as in other patients indicating, that remethylation of methionine from homocysteine was enhanced by betaine.

At least two different biochemical mechanisms may account for the rise in met/tHcy. First, the direct effect of dietary methionine restriction in C $\beta S$ deficiency is a decrease in the "available pool of methionine" and a secondary decrease in production of homocysteine. ${ }^{16}$ Second, increasing the alternate pathway for remethylation of homocysteine is expected to increase methionine by remethylating homocysteine. The mean ratio of methionine to total plasma homocysteine increased almost an order of magnitude (0.65 to 6.1) and provided metabolic evidence supporting the "rerouting" as a major metabolic fate of homocysteine. Without flux studies, we cannot determine whether this rise in met/tHcy was partially due to the reduction in the overall "available methionine pool" and the secondary drop in homocysteine. However, because in Patient 4 methionine was lower on betaine, than off betaine, yet his met/tHcy increased, it is unlikely that reduced methionine pools played a major role in his response.

Molecular analysis of the $C \beta S$ gene confirmed the clinical assessment of B6 response in C $\beta S$ deficiency by identifying specific gene mutations (genotypes) and enabled genotype/ phenotype correlation. The interpretation of the $C \beta S$ genotype-phenotype relationship includes prediction of an individual's response to treatment with B6 (pyridoxine), betaine, and/or a methionine-restricted, cystine supplemented diet. For example, responsiveness to B6 (pyridoxine) supplementation was reported for I278T homozyotes. ${ }^{11}$ However, there is residual enzyme activity with minimal growth in yeast functional assays ${ }^{31}$ for homozygotes, but not for compound het- erozygotes with other mutant alleles. In our study when the I278T mutation was present with either D376N or L101P, the patient was B6-nonresponsive. Interpretation of genotypephenotype correlation is complex and must include both the environment (i.e., methionine intake) and undefined epigenetic metabolism. For example, T262M and L101P are classified as mixed response because the same genotype was found in both B6-responsive and B6-nonresponsive phenotypes. ${ }^{11,21}$ Although we classified all our patients as B6-nonresponsive based on metabolic response, different mutations may alter the effect of betaine on plasma met/tHcy levels. Two patients associated with V320A and Q526K mutations, reported as nonresponsive to B6 supplementation, ${ }^{11}$ and responded more efficiently to betaine treatment when compared to the three patients associated with the $\mathrm{B} 6$ responsive or mixed response genotype categories. In functional yeast assays, the V320A mutation enabled growth in these cysteine auxotrophs in response to B6 supplementation. ${ }^{31}$ This data suggests that defining genotypes will assist in predicting response to betaine therapy. Studies of more patients with B6 nonresponsive mutations will assist in further understanding the genotype-phenotype relationship. Because phenotypes associated with $C \beta S$ genotypes are still unclear, the complexity of genotype/phenotype correlations will require further knowledge of alternate homocysteine metabolism (epigenes) as well as nutritional exposure (environment) using multivariate analysis.

Despite varying compliance and unknown variables in this prospective study, the use of betaine by patients with homocystinuria, classified clinically as B6-nonresponsive, improved metabolic control. This improvement was achieved in 3 to 6 months, without either side-effect or adverse reactions, consistent with previous reports. ${ }^{9}, 13,27,28$ Most importantly, betaine therapy was associated with the normalization of tHcy in $3 / 5$ patients whose genotypes had some residual C $\beta S$ activity. The importance of a low methionine diet to betaine's therapeutic effect has been suggested ${ }^{34}$; however, this is the first report of the efficacy of betaine treatment when administered in conjunction with dietary methionine restriction to reduce tHcy in persons with B6-nonresponsive C $\beta$ S deficiency.

\section{ACKNOWLEDGMENTS}

This study was supported in part by USPHS research grants no. M01-RR00039, to Emory University for a General Clinical Research Center, and grant nos. 1K23-RR15530-01 to M.T.S., and HL57299-01 to W.D.K. We dedicate this manuscript to our colleague Mark T. Steen, MD, PhD who initiated this clinical study.

\section{References}

1. Gaustadnes M, Ingerslev J, Rutiger N. Prevalence of congenital homocystinuria in Denmark. (letter) N Engl J Med 1999;340:1513.

2. Mudd SH, Levy HL, Skovby F. Disorders of Transsulfuration. In: Scriver CR, Beau$\operatorname{det}$ A, Sly W, Valle D, editors. The Metabolic basis of inherited disease. New York: McGraw-Hill, 1995:693-734.

3. Kraus JP, Packman S, Fowler B, Rosenberg LE. Purification and properties of cystathionine beta-synthase from human liver. Evidence for identical subunits. J Bio Chem 1978;253:6523-6528. 
4. Kery V, Bukovska G, Kraus JP. Transsulfuration depends on heme in addition to pyridoxal 5'-phosphate. Cystathionine beta-synthase is a heme protein. J Biol Chem 1994;269:25283-25288.

5. Dudman NP, Guo XW, Gordon RB, Dawson PA, Wilcken DEL. Human homocysteine catabolism: three major pathways and their relevance to the development of arterial occlusive disease. J Nutr 1996;126:295S-300S.

6. Nygaard O, Nordrehaug JE, Refsum H, Ueland PM, Farstad M, Vollset SE. Plasma homocysteine levels and mortality in patients with coronary artery disease. $N$ Engl J Med 1997;337:230-236.

7. Graham IM, Daly LE, Refsum HM, Robinson K, Brattstrom LE, Ueland PM et al. Plasma homocysteine as a risk factor for vascular disease. The European Concerted Action Project. J AMA 1997;277:1775-81.

8. Mudd SH, Skovby F, Levy HL, Skovby F, Levy HL, Pettigrew KD et al. The natural history of homocystinuria due to cystathionine beta-synthase deficiency. Am J Hum Genet 1985;37:1-31.

9. Wilcken DEL, Wilcken B. The natural history of vascular disease in homocystinuria and the effects of treatment. J Inher Metab Dis 1997;20:295-300.

10. Yap S, Naughten E. Homocystinuria due to cystathionine beta-synthase deficiency in Ireland: 25 years' experience of a newborn screened and treated population with reference to clinical outcome and biochemical control. J Inherit Metab Dis 1998; $738-747$.

11. Kraus JP, Janosik M, Kozich V, Mandell R, Shih V, Sperandeo G et al. Cystathionine beta-synthase mutations in homocystinuria. Hum Mutat 1999;13:362-375.

12. Storch KJ, Wagner DA, Young VR. Methionine kinetics in adult men: effects of dietary betaine on L- $\left[{ }^{2} \mathrm{H}_{3}\right.$-methyl $\left.1-{ }^{13} \mathrm{C}\right]$ methionine. Am J Clin Nutr 1991;54:38694.

13. Wilcken DEL, Wilcken B, Dudman NPB, Tyrrell PA. Homocystinuria: The effects of betaine in the treatment of patients not responsive to pyridoxine. $N \mathrm{Engl} \mathrm{J} \mathrm{Med}$ 1983;309:448-453.

14. Surtees R, Bowron A, Leonard J. Cerebrospinal fluid and plasma total homocysteine and related metabolites in children with cystathionine beta-synthase deficiency: the effect of treatment. Peds Res 1997;42:577-582.

15. Kishi T, Kawamura I, Harada Y, Eguchi T, Sakura N, Ueda K et al. Effect of betaine on S-adenosylmethionine levels in the cerebrospinal fluid in a patient with methylenetetrahydrofolate reductase deficiency and peripheral neuropathy. J Inh Metab Dis 1994;17:560-565.

16. Elsas LJ, Acosta PB. Nutrition support of inherited metabolic diseases. In: Shils M, Olson J, Shike M, Ross A, editors. Diet and Nutrition in the prevention and treatment of disease, in Modern Nutrition in Health and Disease, 9th ed. Baltimore: Williams \& Wilkins, 1998:1337-1379.

17. Boddie AM, Steen MT, Sullivan KM, Coates RJ, Pasquali M, Dembure PP et al. Low plasma folate and total plasma homocysteine in heterozygotes for cystathionine beta-synthase deficiency. Metabolism 1998;47:207-211.

18. Steen MT, Boddie AM, Fisher AJ, Sullivan KM, MacMahon W, Saxe D et al. NeuralTube defects are associated with low concentrations of cobalamin in amniotic fluid. Prenat Diag 1998;18:545-555.

19. Kraus JP, Oliveriusova J, Sokolova J, Kraus E, Vlcek C, de Franchis R et al. The human cystathionine beta-synthase gene: complete sequence, alternative splicing, and polymorphisms. Genomics 1998;52:312-324.
20. Kruger WD, Wang L, Jhee KH, Singh RH, Pasquali M, Elsas LJ. Cystathionine $\beta$-synthase deficiency in the Southeastern United States: Correlation of clinical and biochemical phenotype with genotype. Hum Mutat 2003;22:434-441.

21. Kruger WD, Cox DR. A yeast system for expression of human cystathionine betasynthase: structural and functional conservation of the human and yeast genes. Proc Natl Acad Sci U S A 1994;91:6614-6618.

22. Gallagher PM, Naughten E, Hanson NQ, Schwichtenberg K, Bignell M, Yuan M et al. Characterization of mutations in the cystathionine beta-synthase gene in Irish patients with homocystinuria. Mol Genet Metab 1998;65:298-302.

23. Kluijtmans LAJ, Boers GHJ, Kraus JP, van den Heuvel LPWJ, Cruysberg JRM, Trijbels FJM et al. The molecular basis of cystathionine beta-synthase deficiency in Dutch patients with homocystinuria: effect of CBS genotype on biochemical and clinical phenotype and on response to treatment. Am J Hum Genet 1999;65:59-67.

24. Kim CE, Gallagher PM, Gottormsen AB, Refsum H, Ueland PM, Ose L et al. Functional modeling of vitamin-responsiveness in yeast: a common pyridoxine-responsive cystathionine beta-synthase mutation in homocystinuria. Hum Mol Genet 1997; 2213-2221.

25. Dawson PA, Cox AJ, Emmerson BT, Dudman NP, Dudman NP, Kraus JP et al Characterisation of five missense mutations in the cystathionine bet-synthase gene from three patients with B6-nonresponsive homocystinuria. Eur J Hum Genet 1997; 5:15-21.

26. Trondle U, Sunder-Plassmann G, Burgmann H, Buchmayer H, Kramer L, Bieglmayer $\mathrm{C}$ et al. Molecular and clinical characterization of homocystinuria in two Austrian families with cystathionine beta-synthase deficiency. Acta Med Austriaca 2001;28:145-151.

27. Wilcken DEL, Dudman NP, Tyrrell PA. Homocystinuria - the effects of betaine in the treatment of pyridoxine-responsive patients. Metabolism 1985;34:1115-1121.

28. Walter JH, Wraith JE, White FJ, Bridge C, Till J. Strategies for the treatment of cystathionine beta-synthase deficiency: the experience of the Willink Biochem Genet Unit over the past 30 years. Eur J Pediatr 1998;157:S71-S76.

29. Smolin LA, Benevenga NJ, Berlow S. The use of betaine for the treatment of homocystinuria. J Pediatr 1981;99:467-472.

30. Shan X, Kruger WD. Correction of disease-causing CBS mutations in yeast. Nat Genet 1998;19:91-93.

31. Gaustadnes M, Wilcken B, Oliveriusova J, McGill J, Fletcher J, Kraus JP et al. The molecular basis of cystathionine Beta-synthase deficiency in Australian patients: Genotype-phenotype correlations and response to treatment. Hum Mutat 2002;20: $117-126$.

32. Shih VE, Fringer JM, Mandell R, Kraus JP, Berry GT, Heidenreich RA et al. A missense mutation (I278T) in the cystathionine beta-synthase gene prevalent in pyridoxine-responsive homocystinuria and associated with mild clinical phenotype. Am J Hum Genet 1995;57:34-39.

33. Gallagher PM, Naughten E, Hanson NQ, Schwichtenberg K, Bignell M, Yuan M et al. Characterization of mutations in the cystathionine beta-synthase gene in Irish patients with homocystinuria. Mol Genet Metab 1998;65:298-203.

34. Sakamoto A, Sakura N. Limited effectiveness of betaine therapy for cystathionine beta synthase deficiency. Pediatr Internat 2003;45:333-338. 